\title{
Superconvergence of the Iterated Galerkin Methods for Hammerstein Equations
}

\author{
Hideaki Kaneko \\ Department of Mathematics and Statistics \\ Old Dominion University \\ Norfolk, Virginia 23529-0077, USA \\ and \\ Yuesheng $\mathrm{Xu}^{*}$ \\ Department of Mathematics \\ North Dakota State University \\ Fargo, North Dakota 58105, USA
}

${ }^{*}$ This author is partially supported by NASA under grant NAG3-1312 


\title{
SUPERCONVERGENCE OF THE ITERATED GALERKIN METHODS FOR HAMMERSTEIN EQUATIONS
}

\section{H. Kaneko and Y. Xu}

\begin{abstract}
In this paper, the well known iterated Galerkin method and iterated Galerkin-Kantorovich regularization method for approximating the solution of Fredholm integral equations of the second kind are generalized to Hammerstein equations with smooth and weakly singular kernels. The order of convergence of the Galerkin method and those of superconvergence of the iterated methods are analyzed. Numerical examples are presented to illustrate the superconvergence of the iterated Galerkin approximation for Hammerstein equations with weakly singular kernels.
\end{abstract}

Key words: the iterated Galerkin method, the iterated Galerkin-Kantorovich regularization, Hammerstein equations with weakly singular kernels, superconvergence.

Mathematics Subject Classification (1990): 65B05, 45L10.

\section{Introduction}

In this paper, we consider the following Hammerstein equation

$$
x(t)-\int_{0}^{1} k(t, s) \psi(s, x(s)) d s=f(t), \quad 0 \leq t \leq 1,
$$

where $k, f$ and $\psi$ are known functions and $x$ is the function to be determined. Define $k_{t}(s) \equiv$ $k(t, s)$ for $t, s \in[0,1]$ to be the $t$ section of $k$. We assume throughout this paper unless stated otherwise, the following conditions on $k, f$ and $\psi$ :

1. $\lim _{t \rightarrow \tau}\left\|k_{t}-k_{\tau}\right\|_{\infty}=0, \quad \tau \in[0,1]$

2. $M \equiv \sup _{t} \int_{0}^{1}|k(t, s)| d s<\infty$;

3. $f \in C[0,1]$;

4. $\psi(s, x)$ is continuous in $s \in[0,1]$ and Lipschitz continuous in $x \in(-\infty, \infty)$, i.e., there exists a constant $C_{1}>0$ for which

$$
\left|\psi\left(s, x_{1}\right)-\psi\left(s, x_{2}\right)\right| \leq C_{1}\left|x_{1}-x_{2}\right|, \text { for all } x_{1}, x_{2} \in(-\infty, \infty)
$$


5. the partial derivative $\psi^{(0,1)}$ of $\psi$ with respect to the second variable exists and is Lipschitz continuous, i.e., there exists a constant $C_{2}>0$ such that

$$
\left|\psi^{(0,1)}\left(t, x_{1}\right)-\psi^{(0,1)}\left(t, x_{2}\right)\right| \leq C_{2}\left|x_{1}-x_{2}\right|, \text { for all } x_{1}, x_{2} \in(-\infty, \infty)
$$

6. for $x \in C[0,1], \psi(., x()),. \psi^{(0,1)}(., x().) \in C[0,1]$.

Additional assumptions will be given in Sections 2, 3 and 4 when they are needed.

Numerical methods for approximating the solutions of the Hammerstein equations have been studied extensively in the literature. A variation of Nyström's method was proposed by Lardy (1981). A new collocation type method was presented by Kumar and Sloan (1987) and its superconvergence properties were obtained by Kumar (1987). Two different discrete collocation methods were proposed by Kumar (1988) and Atkinson and Flores (1991). A degenerate kernel scheme was intoduced by Kaneko and Xu (1991) for equations (1.1) with smooth kernels. A product integration method and a collocation method were used to solve Hammerstein equations with weakly singular kernels, and certain superconvergence properties of the approximate solutions were discovered by Kaneko, Noren and Xu (1992). A nice review paper by Atkinson (1992) is recommended to the readers who require more information on the numerical treatments of Hammerstein equations. Some theoretical results about Hammerstein equations may be found in a book by Zeidler (1990). The purpose of this paper is to investigate the superconvergence property of the iterated Galerkin method and iterated Kantorovich Galerkin method for the solution of the Hammerstein equation (1.1). The iterated method may be viewed as a nonlinear transformation (iteration) that accelerates the convergence of the approximate solutions obtained from the Galerkin approximation. The general theory of the acceleration of convergence of a sequence by linear or nonlinear transformations was studied by Wimp (1981) and Delahaye (1988), and in the references cited there.

For the Fredholm integral equations of the second kind, the Galerkin and the iterated Galerkin methods have been investigated by many authors, e.g., see Graham (1982), Graham, Joe and Sloan (1985), Sloan (1976), Sloan (1990), Sloan and Thomee (1985) and Vainikko, Pedas and Uba (1984). In these papers that deal with the iterated Galerkin method, it has been shown that under some suitable conditions the iterated Galerkin method gives a rate of convergence that is faster than the rate obtainable by the Galerkin method, a phenomenon commonly known as superconvergence.

The order of convergence for Galerkin approximation for the solutions of Hammerstein equations with weakly singular kernels can be obtained by a direct extension of the corresponding 
result in the Fredholm case. However, it does not seem to be available in the literature. Hence, we include the results in Section 2 for completeness. A substantial number of proofs of the theorems in Section 2 will be omitted since they are straightforward and follow from the work of Vainikko (1967) and from that of Atkinson and Potra (1987). In the latter paper, the reader can find the general theory of the Galerkin and the iterated Galerkin methods for the equation $x=\mathcal{K} x$ where $\mathcal{K}$ is a completely continuous operator of a domain in a Banach space into itself. Our present approach and results are different from those of Atkinson and Potra (1987) in a number of ways. For instance, we establish an estimate of improvement that we can expect when the iterated Galerkin scheme is applied to the weakly singular Hammerstein equations. This will be done in Section 3. Several related results on superconvergence are also established in Section 3. In Section 3, we deal with equations with weakly singular kernels and "nice" forcing terms, while in Sections 4, we tackle equations with both singular kernels and singular forcing terms by employing the classical Kantorovich regularization technique. We extend the results of the iterated Galerkin method to the iterated Galerkin-Kantorovich regularization method. Numerical examples are given in Section 5 to illustrate the theoretical estimates.

\section{The Galerkin Methods for Hammerstein Equations}

In this section, we develop the Galerkin method for Hammerstein equations and establish the order of convergence. Results concerning the Galerkin approximation using spline functions for the solutions of equation (1.1) with smooth and weakly singular kernels are presented.

Let $n$ be a positive integer and $\left\{X_{n}\right\}$ be a sequence of finite dimensional subspaces of $C[0,1]$ such that for any $x \in C[0,1]$ there exists a sequence $\left\{x_{n}\right\}, x_{n} \in X_{n}$, for which

$$
\left\|x_{n}-x\right\|_{\infty} \rightarrow 0 \text { as } n \rightarrow \infty
$$

Let $P_{n}: L_{2}[0,1] \rightarrow X_{n}$ be an orthogonal projection for each $n$. We assume that the projection $P_{n}$ when restricted to $C[0,1]$ is uniformly bounded, i.e.

$$
P:=\sup _{n}\left\|\left.P_{n}\right|_{C[0,1]}\right\|_{\infty}<\infty
$$

Then from (2.1) and (2.2), it follows that for each $x \in C[0,1]$,

$$
\left\|P_{n} x-x\right\|_{\infty} \rightarrow 0, \text { as } n \rightarrow \infty .
$$

Now let

$$
(K \Psi)(x)(t) \equiv \int_{0}^{1} k(t, s) \psi(s, x(s)) d s
$$


With this notation, equation (1.1) takes the following operator form

$$
x-K \Psi x=f .
$$

In many interesting cases, equation (1.1) allows multiple solutions. Hence it is assumed for the remaining of this paper that we are treating a solution $x_{0}$ of equation (1.1) that is isolated.

Let $\left\{\varphi_{n j}\right\}_{j=1}^{n}$ be a set of linearly independent functions that spans $X_{n}$. The Galerkin method is to find

$$
x_{n}=\sum_{j=1}^{n} b_{n j} \varphi_{n j}
$$

that satisfies

$$
x_{n}-P_{n} K \Psi x_{n}=P_{n} f .
$$

Equivalently one is required to find $b_{n j}$ 's that satisfy the system of nonlinear equations described by

$$
\sum_{j=1}^{n} b_{n j}<\varphi_{n j}, \varphi_{n i}>-<\int_{0}^{1} k(t, s) \psi\left(s, \sum_{j=1}^{n} b_{n j} \varphi_{n j}(s)\right) d s, \varphi_{n i}>=<f, \varphi_{n i}>, 1 \leq i \leq n,
$$

where $<., .>$ denotes the inner product in $L_{2}$.

We next estimate the error of the Galerkin approximate solutions to the exact solutions. For notation convenience, we introduce operators $\hat{T}$ and $T_{n}$ by letting

$$
\hat{T} x \equiv f+K \Psi x
$$

and

$$
T_{n} x_{n} \equiv P_{n} f+P_{n} K \Psi x_{n}
$$

so that equations (2.4) and (2.5) can be written respectively as $x=\hat{T} x$ and $x_{n}=T_{n} x_{n}$. A proof of the following theorem can be made by directly applying Theorem 2 of Vainikko (1967). The paper of Atkinson and Potra (1987) is also useful in this connection.

Theorem 2.1 Let $x_{0} \in C[0,1]$ be an isolated solution of equation (2.4). Assume that 1 is not an eigenvalue of the linear operator $(K \Psi)^{\prime}\left(x_{0}\right)$, where $(K \Psi)^{\prime}\left(x_{0}\right)$ denotes the Fréchet derivative of $K \Psi$ at $x_{0}$. Then the Galerkin approximation equation (2.5) has a unique solution $x_{n} \in B\left(x_{0}, \delta\right)$ for some $\delta>0$ and for sufficiently large $n$. Moreover, there exists a constant $0<q<1$, independent of $n$, such that

$$
\frac{\alpha_{n}}{1+q} \leq\left\|x_{n}-x_{0}\right\|_{\infty} \leq \frac{\alpha_{n}}{1-q},
$$

where $\alpha_{n} \equiv\left\|\left(I-T_{n}^{\prime}\left(x_{0}\right)\right)^{-1}\left(T_{n}\left(x_{0}\right)-\hat{T}\left(x_{0}\right)\right)\right\|_{\infty}$. Finally,

$$
E_{n}\left(x_{0}\right) \leq\left\|x_{n}-x_{0}\right\|_{\infty} \leq C E_{n}\left(x_{0}\right),
$$


where $C$ is a constant independent of $n$ and $E_{n}\left(x_{0}\right)=\inf _{u \in X_{n}}\left\|x_{0}-u\right\|_{\infty}$.

We denote by $W_{p}^{m}[0,1], 1 \leq p \leq \infty$, the Sobolev space of functions $g$ whose $m$-th generalized derivative $g^{(m)}$ belongs to $L_{p}[0,1]$. The space $W_{p}^{m}[0,1]$ is equipped with the norm

$$
\|g\|_{W_{p}^{m}} \equiv \sum_{k=0}^{m}\left\|g^{(k)}\right\|_{p}
$$

We now specify the finite dimensional subspace $X_{n}$. For any positive integer $n$, let

$$
\Pi_{n}: 0=t_{0}<t_{1}<\ldots<t_{n-1}<t_{n}=1
$$

be a partition of $[0,1]$. Let $r$ and $\nu$ be nonnegative integers satisfying $0 \leq \nu<r$. Let $S_{r}^{\nu}\left(\Pi_{n}\right)$ denote the space of splines of order $r$, continuity $\nu$, with knots at $\Pi_{n}$, that is

$$
S_{r}^{\nu}\left(\Pi_{n}\right)=\left\{x \in C^{\nu}[0,1]:\left.x\right|_{\left[t_{i}, t_{i+1}\right]} \in \mathcal{P}_{r-1}, \text { for each } i=0,1, \ldots, n-1\right\}
$$

where $\mathcal{P}_{r-1}$ denotes the space of polynomials of degree $\leq r-1$. We assume that the sequence of partitions $\Pi_{n}$ of $[0,1]$ satisfies the condition that there exists a constant $C>0$, independent of $n$, with the property:

$$
\frac{\max _{1 \leq i \leq n}\left(t_{i}-t_{i-1}\right)}{\min _{1 \leq i \leq n}\left(t_{i}-t_{i-1}\right)} \leq C, \text { for all } n .
$$

It is known from de Boor (1976) and Douglas, Dupont and Wahlbin (1975) that condition (2.14) implies that the Galerkin projections $P_{n}$ are uniformly bounded. In addition, it is also well known from Demko (1976) and De Vore (1976) that if $0 \leq \nu<r, 1 \leq p \leq \infty, m \geq 0$ and $x \in W_{p}^{m}$, then for each $n \geq 1$, there exists $u_{n} \in S_{r}^{\nu}\left(\Pi_{n}\right)$ such that

$$
\left\|x-u_{n}\right\|_{p} \leq C h^{\mu}\|x\|_{W_{p}^{\mu}}
$$

where $\mu=\min \{m, r\}$ and $h=\max _{1 \leq i \leq n}\left(t_{i}-t_{i-1}\right)$. Using Theorem 2.1 and the inequalities (2.12) and (2.15), we obtain the following theorem.

Theorem 2.2 Let $x_{0}$ be an isolated solution of equation (1.1) and let $x_{n}$ be the solution of equation (2.5) in a neighborhood of $x_{0}$. Assume that 1 is not an eigenvalue of $(K \Psi)^{\prime}\left(x_{0}\right)$. If $x_{0} \in W_{\infty}^{l}(0 \leq l \leq r)$, then

$$
\left\|x_{0}-x_{n}\right\|_{\infty}=O\left(h^{\mu}\right)
$$

where $\mu=\min \{l, r\}$. If $x_{0} \in W_{p}^{l}(0<l \leq r, 1 \leq p<\infty)$, then

$$
\left\|x_{0}-x_{n}\right\|_{\infty}=O\left(h^{\nu}\right)
$$

where $\nu=\min \{l-1, r\}$. 
We remark that a similar result of Galerkin's method for Urysohn equations was obtained by Atkinson and Potra (1987). Hence, Theorem 2.2 may be derived by specializing their result to Hammerstein equations.

In the remaining portion of this section, we investigate the order of convergence of the Galerkin method for Hammerstein equations with weakly singular kernels. For this purpose, we define some necessary notation. For any $\epsilon \in R$, let $[0,1]_{\epsilon}=\{t \in[0,1]: t+\epsilon \in[0,1]\}$. Let $\Delta_{h}$ denote the forward difference operator with step size $h$. For $\alpha>0$ and $1 \leq p \leq \infty$, we define the Nikol'skii space $N_{p}^{\alpha}[0,1]$ by

$$
N_{p}^{\alpha}[0,1]=\left\{x \in L_{p}[0,1]:|x|_{\alpha, p}:=\sup _{h \neq 0} \frac{1}{|h|^{\alpha_{0}}}\left\|\Delta_{h}^{2} x^{[\alpha]}\right\|_{L_{p}[0,1]_{2 h}}<\infty\right\},
$$

where $[\alpha]$ is an integer and $0<\alpha_{0} \leq 1$ are chosen so that $\alpha=[\alpha]+\alpha_{0}$. Clearly, $N_{p}^{\alpha}[0,1]$ is a Banach space with the norm $\|x\|_{\alpha, p}=\|x\|_{p}+|x|_{\alpha, p}$. We remark that the function $t^{\alpha-1}$ is in $N_{1}^{\alpha}[0,1]$ but is not in $N_{1}^{\beta}[0,1]$, for any $\beta>\alpha$, and $\log t \in N_{1}^{1}[0,1]$. It is known from Graham (1982) that

$$
N_{p}^{m+\epsilon}[0,1] \subseteq W_{p}^{m}[0,1] \subseteq N_{p}^{m}[0,1] \subseteq N_{p}^{m-\epsilon}[0,1],
$$

for $m \in N, 0<\epsilon<1$, and $1 \leq p \leq \infty$; and

$$
N_{p}^{\alpha}[0,1] \subseteq N_{q}^{\beta}[0,1]
$$

for $\alpha>0,1 \leq p \leq q \leq \infty$ and $\beta=\alpha-(1 / p-1 / q)>0$. We consider Hammerstein equations with kernels given by

$$
k(t, s)=m(t, s) k(t-s), t, s \in[0,1]
$$

with $k \in N_{1}^{\alpha}[0,1]$ for some $0<\alpha<1$ and $m \in C^{2}([0,1] \times[0,1])$, and $\psi$ as defined in the previous section.

Again, we let $X_{n}=S_{r}^{\nu}\left(\Pi_{n}\right)$. When no further conditions are made on the partition $\Pi_{n}$ other than the one given by (2.14), the next theorem gives the best possible order of convergence of the Galerkin approximation to the solution of equation (1.1) with a weakly singular kernel defined by $(2.19)$.

Theorem 2.3 Let $x_{0}$ be an isolated solution of equation (1.1) with a kernel given by (2.19). Assume that 1 is not an eigenvalue of $(K \Psi)^{\prime}\left(x_{0}\right)$. If $f \in N_{1}^{\beta+1}[0,1]$ for some $0<\beta<1$, then

$$
\left\|x_{0}-x_{n}\right\|_{\infty}=O\left(h^{\gamma}\right)
$$

with $\gamma=\min \{\alpha, \beta\}$. 
Proof: By Theorem 2.1, we have

$$
\left\|x_{0}-x_{n}\right\|_{\infty} \leq C \inf _{u \in S_{r, n}^{\nu}\left(\Pi_{n}\right)}\left\|x_{0}-u\right\|_{\infty}
$$

A similar proof to the one given for Theorem 3 (ii) of Graham (1982) shows that if $f \in N_{1}^{\beta+1}[0,1]$ then $x_{0} \in N_{1}^{\min \{\alpha+1, \beta+1\}}[0,1] \subseteq N_{\infty}^{\min \{\alpha, \beta\}}[0,1]$. In addition, (2.17) implies that $f \in W_{1}^{1}[0,1]$. Hence $f$ is equal to an absolutely continuous function almost everywhere. Without loss of generality, we have $f \in W_{1}^{1}[0,1] \cap C[0,1]$. It can be shown that $x_{0} \in C[0,1]$. Thus, $x_{0} \in N_{\infty}^{\gamma}[0,1] \cap C[0,1]$. It was proved in Graham (1982) that if $\phi \in N_{\infty}^{\eta}[0,1] \cap C[0,1]$ for some $0<\eta<1$, then there exists a spline $v \in S_{r}^{\nu}\left(\Pi_{n}\right)$ such that $\|\phi-v\|_{\infty} \leq C h^{\eta}$ where $C$ is a constant independent of $h$. The result of this theorem follows immediately from (2.20) and the above argument.

Now we consider a special form of (2.19). Namely we assume

$$
k(t, s)=m(t, s) g_{\alpha}(|t-s|)
$$

where $m \in C^{\mu+1}([0,1] \times[0,1])$ and

$$
g_{\alpha}(s)= \begin{cases}s^{\alpha-1}, & 0<\alpha<1, \\ \log s, & \alpha=1 .\end{cases}
$$

With these kernels, certain regularities of the solutions of (1.1) are known. Let $S$ be a finite set in $[0,1]$ and we define the function $\omega_{S}(t)=\inf \{|t-s|: s \in S\}$. A function $x$ is said to be of Type $(\alpha, k, S)$, for $-1<\alpha<0$, if

$$
\left|x^{(k)}(t)\right| \leq C\left[\omega_{S}(t)\right]^{\alpha-k} t \notin S,
$$

and for $\alpha>0$, if the above condition holds and $x \in \operatorname{Lip}(\alpha)$. Kaneko, Noren and Xu (1990) proved that if $f$ is of $\operatorname{Type}(\beta, \mu,\{0,1\})$, then a solution of equation (1.1) is of Type $(\gamma, \mu,\{0,1\})$, where $\gamma=\min \{\alpha, \beta\}$. In order to recover the optimal rate of convergence of numerical solutions, we define a partition $\Pi_{n}^{\gamma}$ of $[0,1]$ corresponding to the regularity of a solution. The knots of this partition $\Pi_{n}^{\gamma}$ are given by

$$
\begin{array}{ll}
t_{i}=(1 / 2)(2 i / n)^{q}, & 0 \leq i \leq n / 2, \\
t_{i}=1-t_{n-i}, & n / 2<i \leq n,
\end{array}
$$

where $q=\frac{r}{\gamma}$. Let $S_{r, n}^{\nu, \gamma}=S_{r}^{\nu}\left(\Pi_{n}^{\gamma}\right)$, with $r=1$ and $\nu=0$, or $r \geq 2$ and $\nu \in\{0,1\}$. The following theorem gives the order of convergence of the Galerkin approximations to the solution of Hammerstein equations with kernels defined by (2.21) and (2.22). It should be noted that the technique of approximating a solution of the type described above by elements from the nonlinear spline space has been used on many occasions in dealing with the weakly singular Fredholm 
integral equations. For example, Vainikko and Uba (1981) describe the collocation method, whereas in Vainikko, Pedas and Uba (1984) they describe the Galerkin method. Schneider (1981) on the other hand establishes the product-integration method based upon the idea of the nonlinear spline approximation with nonuniform knots.

Theorem 2.4 Let $x_{0}$ be an isolated solution of (1.1) with kernels (2.21) and (2.22) and let $x_{n}$ be the Galerkin approximation to $x_{0}$. Let $m \in C^{\mu+1}([0,1] \times[0,1])$, and $f$ be of Type $(\beta, \mu,\{0,1\})$. Assume that $\psi \in C^{(0,1)}([0,1] \times(-\infty, \infty))$ for $\mu=0,1$ and $\psi \in C^{\mu-1}([0,1] \times(-\infty, \infty))$ for $\mu \geq 2$. We also assume 1 is not an eigenvalue of $(K \Psi)^{\prime}\left(x_{0}\right)$. Then

$$
\left\|x_{0}-x_{n}\right\|_{\infty}=O\left(\frac{1}{n^{r}}\right)
$$

Proof: This follows from Theorem 2.1, the regularity of the solution $x_{0}$, and from the results of Rice (1969).

\section{The Iterated Galerkin Method}

In this section, we study the superconvergence of the iterated Galerkin method for the Hammerstein equation (1.1). Generalizing the linear case we first define the iterated scheme. Assume that $x_{0}$ is an isolated solution of (1.1). As in Section 2, let $P_{n}$ be the orthogonal projection from $L_{2}[0,1]$ onto $X_{n}$ with conditions (2.1) and (2.2) satisfied. Assume that $x_{n}$ is the unique solution of $(2.5)$ in the sphere $B\left(x_{0}, \delta\right)$ for some $\delta>0$. Define

$$
x_{n}^{\prime}=f+K \Psi x_{n}
$$

Applying $P_{n}$ to the both sides of (3.1), we obtain

$$
P_{n} x_{n}^{\prime}=P_{n} f+P_{n} K \Psi x_{n} .
$$

Comparing (3.2) with (2.5), we see that

$$
P_{n} x_{n}^{\prime}=x_{n}
$$

Upon substituting (3.3) into (3.1), we find that the function $x_{n}^{\prime}$ satisfies the following new Hammerstein equation

$$
x_{n}^{\prime}=f+K \Psi P_{n} x_{n}^{\prime} .
$$

By letting $S_{n} \equiv f+K \Psi P_{n}$, we may rewrite (3.4) as $x_{n}^{\prime}=S_{n} x_{n}^{\prime}$. We first study the invertibility of the linear operators $I-S_{n}^{\prime}\left(x_{0}\right)$ in the following theorem, which will be used to prove the main results of this section. 
Lemma 3.1 Let $x_{0} \in C[0,1]$ be an isolated solution of (1.1). Assume that 1 is not an eigenvalue of $(K \Psi)^{\prime}\left(x_{0}\right)$. Then for sufficiently large $n$, the operators $I-S_{n}^{\prime}\left(x_{0}\right)$ are invertible and there exists a constant $L>0$ such that

$$
\left\|\left(I-S_{n}^{\prime}\left(x_{0}\right)\right)^{-1}\right\|_{\infty} \leq L, \text { for sufficeintly large } n
$$

Proof: Recalling the definition of Fréchet derivatives $S_{n}^{\prime}\left(x_{0}\right)$ and $\hat{T}^{\prime}\left(x_{0}\right)$, we have, for each $x \in C[0,1]$,

$$
\begin{aligned}
\left\|\hat{T}^{\prime}\left(x_{0}\right)(x)-S_{n}^{\prime}\left(x_{0}\right)(x)\right\|_{\infty} \leq & \sup _{0 \leq t \leq 1} \int_{0}^{1}|k(t, s)| \psi^{(0,1)}\left(s, x_{0}(s)\right) \mid d s\left\|x-P_{n} x\right\|_{\infty} \\
& +C \sup _{0 \leq t \leq 1} M\left\|P_{n}\right\|_{\infty}\|x\|_{\infty}\left\|x_{0}-P_{n} x_{0}\right\|_{\infty} .
\end{aligned}
$$

By (2.1), the last two terms can be made arbitrarily small as $n \rightarrow \infty$. This implies that $S_{n}^{\prime}\left(x_{0}\right) \rightarrow$ $\hat{T}^{\prime}\left(x_{0}\right)$ pointwise in $C[0,1]$, as $n \rightarrow \infty$. By Assumptions 1,2 , and $6, \hat{T}^{\prime}\left(x_{0}\right)$ is a compact operator in $C[0,1]$. Notice that by Assumptions 5,6 and condition $(2.1)$, there exists a constant $C>0$ such that

$$
\left|\psi^{(0,1)}\left(s, P_{n} x_{0}(s)\right)\right| \leq C_{2}\left\|P_{n} x_{0}-x_{0}\right\|_{\infty}+\left\|\psi^{(0,1)}\left(., x_{0}(.)\right)\right\|_{\infty} \leq C, \text { for all } n \text {. }
$$

Therefore, $\left\|S_{n}^{\prime}\left(x_{0}\right)(x)\right\|_{\infty} \leq M C P\|x\|_{\infty}$, and

$$
\left|S_{n}^{\prime}\left(x_{0}\right)(x)(t)-S_{n}^{\prime}\left(x_{0}\right)(x)\left(t^{\prime}\right)\right| \leq C P\left\|k_{t}-k_{t^{\prime}}\right\|_{1}\|x\|_{\infty} .
$$

This implies that $\left\{S_{n}^{\prime}\left(x_{0}\right)\right\}$ is collectively compact. It follows from the theory of collectively compact operators in Anselone (1971) and Atkinson (1976) that $\left(I-S_{n}^{\prime}\left(x_{0}\right)\right)^{-1}$ exists for sufficiently large $n$ and there exists a constant $L>0$ such that $\left\|\left(I-S_{n}^{\prime}\left(x_{0}\right)\right)^{-1}\right\| \leq L$ for sufficiently large $n$.

For simplicity, from Lemma 3.1 we assume without loss of generality that $I-S_{n}^{\prime}\left(x_{0}\right)$ is invertible for each $n \geq 1$ and

$$
L=\sup \left\{\left\|\left(I-S_{n}^{\prime}\left(x_{0}\right)\right)^{-1}\right\|_{\infty}: n \geq 1\right\}<\infty .
$$

Throughout the rest of this section, we assume without further mention that $\delta>0$ satisfies $L C_{2} M P \delta<1$ and $\delta_{1}$ is chosen so that $C_{1} M \delta_{1} \leq \delta$. The following lemma establishes that $x_{n}^{\prime}$ defined in (3.1) is a unique solution of (3.4) in some neighborhood of $x_{0}$ and provides an error bound for $x_{n}^{\prime}$ approximating $x_{0}$.

Lemma 3.2 Let $x_{0} \in C[0,1]$ be an isolated solution of equation (1.1) and $x_{n}$ be the unique solution of (2.5) in the sphere $B\left(x_{0}, \delta_{1}\right)$. Assume that 1 is not an eigenvalue of $(K \Psi)^{\prime}\left(x_{0}\right)$. Then 
for sufficiently large $n, x_{n}^{\prime}$ defined by the iterated scheme (3.1) is the unique solution of (3.4) in the sphere $B\left(x_{0}, \delta\right)$. Moreover, there exists a constant $0<q<1$, independent of $n$, such that

$$
\frac{\beta_{n}}{1+q} \leq\left\|x_{n}^{\prime}-x_{0}\right\|_{\infty} \leq \frac{\beta_{n}}{1-q},
$$

where $\beta_{n}=\left\|\left(I-S_{n}^{\prime}\left(x_{0}\right)\right)^{-1}\left[S_{n}\left(x_{0}\right)-\hat{T}\left(x_{0}\right)\right]\right\|_{\infty}$. Finally,

$$
\left\|x_{n}^{\prime}-x_{0}\right\|_{\infty} \leq C E_{n}\left(x_{0}\right)
$$

Proof: This follows easily using Lemma 2.1 and Theorem 2 of Vainikko (1967).

One way to ensure a superconvergence of the iterated Galerkin method is to assume

$$
\left\|\left.(K \Psi)^{\prime}\left(x_{0}\right)\left(I-P_{n}\right)\right|_{C[a, b]}\right\|_{\infty} \rightarrow 0 \quad \text { as } n \rightarrow \infty .
$$

In this case, using the identity (ref. Theorem 2.3 of Atkinson and Potra (1987))

$$
\begin{aligned}
& \left(I-(K \Psi)^{\prime}\left(x_{0}\right)\right)\left(x_{n}^{\prime}-x_{0}\right) \\
& =\left[I-(K \Psi)^{\prime}\left(x_{0}\right)\left(I-P_{n}\right)\right]\left[K \Psi\left(x_{n}\right)-K \Psi\left(x_{0}\right)-(K \Psi)^{\prime}\left(x_{0}\right)\left(x_{n}-x_{0}\right)\right] \\
& -(K \Psi)^{\prime}\left(x_{0}\right)\left(I-P_{n}\right)\left((K \Psi)^{\prime}\left(x_{0}\right)-I\right)\left(x_{n}-x_{0}\right) .
\end{aligned}
$$

we obtain

$$
\begin{aligned}
\left\|x_{n}^{\prime}-x_{0}\right\|_{\infty} \leq & \left\|\left(I-(K \Psi)^{\prime}\left(x_{0}\right)\right)^{-1}\right\|_{\infty}\left\{\left\|I-(K \Psi)^{\prime}\left(x_{0}\right)\left(I-P_{n}\right)\right\|_{\infty}\right. \\
& \times \sup _{0 \leq \theta \leq 1}\left\|(K \Psi)^{\prime}\left(x_{0}+\theta\left(x_{n}-x_{0}\right)\right)-(K \Psi)^{\prime}\left(x_{0}\right)\right\|_{\infty}\left\|x_{0}-x_{n}\right\|_{\infty} \\
& \left.+\left\|(K \Psi)^{\prime}\left(x_{0}\right)\left(I-P_{n}\right)\left((K \Psi)^{\prime}\left(x_{0}\right)-I\right)\left(x_{n}-x_{0}\right)\right\|_{\infty}\right\} .
\end{aligned}
$$

This with (3.5) gives a superconvergence of $x_{n}^{\prime}$ to $x_{0}$. In the next theorem, we establish superconvergence of the iterated Galerkin method in a general setting. In establishing superconvergence of the iterates of the Fredholm equations, many authors assumed the condition $\left\|K\left(I-P_{n}\right)\right\| \rightarrow 0$ as $n \rightarrow \infty$ with $K$ being a compact linear operator (e.g., Theorem 5 of Graham (1982) and Theorem 3.1 of Sloan (1990)). In our current problem, this is equivalent to assuming condition (3.5). However, the next theorem is proved without assumption (3.5). First, we apply the mean-value theorem to $\psi(s, y)$ to conclude

$$
\psi(s, y)=\psi\left(s, y_{0}\right)+\psi^{(0,1)}\left(s, y_{0}+\theta\left(y-y_{0}\right)\right)\left(y-y_{0}\right)
$$

where $\theta:=\theta\left(s, y_{0}, y\right)$ with $0<\theta<1$. The boundedness of $\theta$ is essential for the proof of the next theorem, although it may depend on $s, y_{0}, y$. Let

$$
g\left(t, s, y_{0}, y, \theta\right)=k(t, s) \psi^{(0,1)}\left(s, y_{0}+\theta\left(y-y_{0}\right)\right)
$$




$$
\left(G_{n} x\right)(t)=\int_{0}^{1} g\left(t, s, P_{n} x_{0}(s), P_{n} x_{n}^{\prime}(s), \theta\right) x(s) d s,
$$

and $(G x)(t)=\int_{0}^{1} g_{t}(s) x(s) d s$, where $g_{t}(s)=k(t, s) \psi^{(0,1)}\left(s, x_{0}(s)\right)$.

Theorem 3.3 Let $x_{0} \in C[0,1]$ be an isolated solution of equation (1.1) and $x_{n}$ be the unique solution of (2.5) in the sphere $B\left(x_{0}, \delta_{1}\right)$. Let $x_{n}^{\prime}$ be defined by the iterated scheme (3.1). Assume that 1 is not an eigenvalue of $(K \Psi)^{\prime}\left(x_{0}\right)$. Then, for all $1 \leq p \leq \infty$,

$$
\left\|x_{0}-x_{n}^{\prime}\right\|_{\infty} \leq C\left\{\left\|x_{0}-P_{n} x_{0}\right\|_{\infty}^{2}+\sup _{0 \leq t \leq 1} \inf _{u \in X_{n}}\left\|k(t, .) \psi^{(0,1)}\left(., x_{0}(.)\right)-u\right\|_{q}\left\|x_{0}-P_{n} x_{0}\right\|_{p}\right\},
$$

where $1 / p+1 / q=1$ and $C$ is a constant independent of $n$.

Proof: Note that from equations (1.1) and (3.4) we have

$$
x_{0}-x_{n}^{\prime}=K\left(\Psi x_{0}-\Psi P_{n} x_{n}^{\prime}\right)=K\left(\Psi x_{0}-\Psi P_{n} x_{0}\right)+K\left(\Psi P_{n} x_{0}-\Psi P_{n} x_{n}^{\prime}\right) .
$$

Replacing $y$ by $P_{n} x_{n}^{\prime}$ and $y_{0}$ by $P_{n} x_{0}$ in equation (3.6), the last term of (3.7) can be written as

$$
K\left(\Psi P_{n} x_{0}-\Psi P_{n} x_{n}^{\prime}\right)(t)=\left(G_{n} P_{n}\left(x_{0}-x_{n}^{\prime}\right)\right)(t) .
$$

Equation (3.7) now becomes

$$
x_{0}-x_{n}^{\prime}=K\left(\Psi x_{0}-\Psi P_{n} x_{0}\right)+G_{n} P_{n}\left(x_{0}-x_{n}^{\prime}\right) .
$$

By using condition (1.2) and the fact $0<\theta<1$, we have, for all $x \in C[0,1]$,

$$
\left\|\left(G_{n} x\right)-(G x)\right\|_{\infty} \leq \sup _{0 \leq t \leq 1} \int_{0}^{1}|k(t, s)| d s\|x\|_{\infty}\left(\left\|P_{n} x_{0}-x_{0}\right\|_{\infty}+\left\|P_{n}\right\|_{\infty}\left\|x_{n}^{\prime}-x_{0}\right\|_{\infty}\right) .
$$

Consequently, by assumption (2.1) and Lemma 3.2,

$$
\left\|G_{n}-G\right\|_{\infty} \leq M\left(\left\|P_{n} x_{0}-x_{0}\right\|_{\infty}+P\left\|x_{n}^{\prime}-x_{0}\right\|_{\infty}\right) \rightarrow 0 \text { as } n \rightarrow \infty .
$$

That is, $G_{n} \rightarrow G$ in the norm of $C[0,1]$ as $n \rightarrow \infty$. Moreover, for each $x \in C[0,1]$,

$$
\sup _{0 \leq t \leq 1}\left|\left(G P_{n} x\right)(t)-(G x)(t)\right|=\sup _{0 \leq t \leq 1}\left|\int_{0}^{1} g_{t}(s)\left[P_{n} x(s)-x(s)\right] d s\right| \leq M M_{1}\left\|P_{n} x-x\right\|_{\infty},
$$

where

$$
M_{1}=\sup _{0 \leq t \leq 1}\left|\psi^{(0,1)}\left(t, x_{0}(t)\right)\right|<+\infty .
$$

It follows that $G P_{n} \rightarrow G$ pointwise in $C[0,1]$ as $n \rightarrow \infty$. Again since $P_{n}$ is uniformly bounded, we have for each $x \in C[0,1]$,

$$
\left\|G_{n} P_{n} x-G x\right\|_{\infty} \leq\left\|G_{n}-G\right\|_{\infty}\left\|P_{n}\right\|_{\infty}\|x\|_{\infty}+\left\|G P_{n} x-G x\right\|_{\infty} .
$$


Thus, $G_{n} P_{n} \rightarrow G$ pointwise in $C[0,1]$ as $n \rightarrow \infty$. By Assumptions 2, 5, and 6, we see that there exists a constant $C>0$ such that for all $n$

$$
\left|\psi^{(0,1)}\left(s, P_{n} x_{0}(s)+\theta\left(P_{n} x_{n}^{\prime}(s)-P_{n} x_{0}(s)\right)\right)\right| \leq C_{2}\left\|P_{n} x_{0}-x_{0}\right\|_{\infty}+\theta C_{2} P\left\|x_{n}^{\prime}-x_{0}\right\|_{\infty}+M_{1} \leq C .
$$

By a proof similar to that for Lemma 3.1, we can show that $\left\{G_{n} P_{n}\right\}$ is collectively compact. Since $G=(K \Psi)^{\prime}\left(x_{0}\right)$ is compact and $(I-G)^{-1}$ exists, it follows from the theory of collectively compact operators that $\left(I-G_{n} P_{n}\right)^{-1}$ exists and is uniformly bounded for sufficiently large $n$. By (3.8), we have the following estimate

$$
\sup _{0 \leq t \leq 1}\left|\left(x_{0}-x_{n}^{\prime}\right)(t)\right| \leq C \sup _{0 \leq t \leq 1}\left|K\left(\Psi x_{0}-\Psi P_{n} x_{0}\right)(t)\right| .
$$

Next, we estimate the function $d(t) \equiv\left|K\left(\Psi x_{0}-\Psi P_{n} x_{0}\right)(t)\right|$. Using (3.6) with $y=P_{n} x_{0}$ and $y_{0}=x_{0}$, we obtain, for $0<\theta<1$,

$$
d(t)=\left|\int_{0}^{1} g\left(t, s, x_{0}(s), P_{n} x_{0}(s), \theta\right)\left(x_{0}(s)-P_{n} x_{0}(s)\right) d s\right| .
$$

Note that $\int_{0}^{1} u(s)\left[x_{0}(s)-P_{n} x_{0}(s)\right] d s=0$, for all $u \in X_{n}$. Thus, for all $u \in X_{n}$,

$$
\begin{aligned}
d(t)= & \left|\int_{0}^{1}\left[g\left(t, s, x_{0}(s), P_{n} x_{0}(s), \theta\right)-u(s)\right]\left(x_{0}(s)-P_{n} x_{0}(s)\right) d s\right| \\
\leq & \int_{0}^{1}\left|g\left(t, s, x_{0}(s), P_{n} x_{0}(s), \theta\right)-g_{t}(s)\right| d s\left\|x_{0}-P_{n} x_{0}\right\|_{\infty} \\
& +\left|\int_{0}^{1}\left[g_{t}(s)-u(s)\right]\left(x_{0}(s)-P_{n} x_{0}(s)\right) d s\right| .
\end{aligned}
$$

Now, by condition (1.2), we have

$$
\int_{0}^{1}\left|g\left(t, s, x_{0}, P_{n} x_{0}(s), \theta\right)-g_{t}(s)\right| d s \leq C_{1} \theta \int_{0}^{1}|k(t, s)| d s\left\|x_{0}-P_{n} x_{0}\right\|_{\infty} \leq C_{1} M\left\|x_{0}-P_{n} x_{0}\right\|_{\infty} .
$$

Moreover, for $1 / p+1 / q=1$,

$$
\left|\int_{0}^{1}\left[g_{t}(s)-u(s)\right]\left[x_{0}(s)-P_{n} x_{0}(s)\right] d s\right| \leq\left\|g_{t}-u\right\|_{q}\left\|x_{0}-P_{n} x_{0}\right\|_{p} .
$$

Therefore,

$$
d(t) \leq C_{1} M\left\|x_{0}-P_{n} x_{0}\right\|_{\infty}^{2}+\left\|g_{t}-u\right\|_{q}\left\|x_{0}-P_{n} x_{0}\right\|_{p}, \text { for all } u \in X_{n}
$$

Hence the desired result follows.

In the next two theorems, we consider the case that $X_{n}=S_{r}^{\nu}\left(\Pi_{n}\right)$ where $\Pi_{n}$ is an arbitrary partition of $[0,1]$ satisfying $(2.14)$. First, we consider the case when both the kernels and the solutions of equation (1.1) are smooth. 
Theorem 3.4 Let $x_{0} \in W_{p}^{l}(0<l \leq r)$ be an isolated solution of (1.1), $x_{n}$ be the unique solution of (2.5) in $B\left(x_{0}, \delta_{1}\right)$, and $x_{n}^{\prime}$ be defined by the iterated scheme (3.1). Assume that 1 is not an eigenvalue of $(K \Psi)^{\prime}\left(x_{0}\right)$. Assume that for all $t \in[0,1], k_{t}(.) \psi^{(0,1)}\left(., x_{0}().\right) \in W_{q}^{m}(0 \leq m \leq r)$. Then

$$
\left\|x_{0}-x_{n}^{\prime}\right\|_{\infty}=O\left(h^{\mu+\min \{\mu, \nu\}}\right)
$$

where $\mu=\min \{l, r\}$ and $\nu=\min \{m, r\}$.

Proof: Since the partition $\Pi_{n}$ of $[0,1]$ satisfies condition $(2.14)$, we conclude that

$$
P:=\sup _{n}\left\|P_{n}\right\|_{\infty}<\infty
$$

Hence,

$$
\left\|x_{0}-P_{n} x_{0}\right\|_{p} \leq\left\|x_{0}-P_{n} x_{0}\right\|_{\infty} \leq(1+P) \inf _{u \in S_{r}^{\nu}\left(\Pi_{n}\right)}\left\|x_{0}-u\right\|_{\infty} \leq C h^{\mu} .
$$

In addition,

$$
\sup _{0 \leq t \leq 1} \inf _{u \in S_{r}^{\nu}\left(\Pi_{n}\right)}\left\|k_{t}(.) \psi^{(0,1)}\left(., x_{0}(.)\right)-u\right\|_{q} \leq C h^{\nu} .
$$

The result of this theorem follows from Theorem 3.3 with $X_{n}=S_{r}^{\nu}\left(\Pi_{n}\right)$.

We remark that Theorem 3.4 may be obtained from Theorem 5.2 of Atkinson and Potra (1987), Theorem 3.4 being a special case of Atkinson and Potra's theorem to Hammerstein equations.

In the following theorem, we assume that $k(t, s)$ is a kernel given by $(2.19)$, i.e., $k(t, s)=$ $m(t, s) k(t-s)$, with $k \in N_{1}^{\alpha}[0,1]$ for some $0<\alpha<1$ and $m \in C^{2}([0,1] \times[0,1])$. Also, we assume that $S_{r}^{\nu}\left(\Pi_{n}\right)$ is such that $\nu \geq 1$.

Theorem 3.5 Let $x_{0}$ be an isolated solution of equation (1.1) with kernels given by (2.19), $x_{n}$ be the unique solution of equation (2.5) in $B\left(x_{0}, \delta_{1}\right)$, and $x_{n}^{\prime}$ be defined by iterated scheme (3.1). Assume that 1 is not an eigenvalue of $(K \Psi)^{\prime}\left(x_{0}\right), f \in N_{1}^{\beta+1}[0,1]$ for some $0<\beta<1$, $\psi^{(0,1)}(., x().) \in W_{1}^{1}$ for $x \in W_{1}^{1}$. Then

$$
\left\|x_{0}-x_{n}^{\prime}\right\|_{\infty}=O\left(h^{2 \gamma}\right)
$$

with $\gamma=\min \{\alpha, \beta\}$.

Proof: Following the proof of Theorem 3.4, we have

$$
\left\|x_{0}-P_{n} x_{0}\right\|_{\infty} \leq(1+P) \inf _{u \in S_{r}^{\nu}\left(\Pi_{n}\right)}\left\|x_{0}-u\right\|_{\infty}
$$


As stated in the proof of Theorem 2.4, we know that

$$
x_{0} \in N_{\infty}^{\gamma}[0,1] \cap C[0,1] \cap W_{1}^{1} .
$$

Using (3.9) and an argument similar to the one used in the proof of Theorem 2.4, we obtain $\| x_{0}-$ $P_{n} x_{0} \|_{\infty} \leq C h^{\gamma}$. Now, by Theorem 4(i) of Graham (1982), we find that there exists $v_{t} \in S_{r}^{\nu}\left(\Pi_{n}\right)$ such that $\left\|k_{t}-v_{t}\right\|_{1}=O\left(h^{\alpha}\right)$. Since $\nu \geq 1$, it follows that $S_{r}^{\nu}\left(\Pi_{n}\right) \subset W_{1}^{1}$. Thus, $v_{t} \in W_{1}^{1}$. From (3.10), $x_{0} \in W_{1}^{1}$. This yields that $\psi^{(0,1)}\left(., x_{0}().\right) \in W_{1}^{1}$. Consequently, $v_{t}(.) \psi^{(0,1)}\left(., x_{0}().\right) \in W_{1}^{1}$. The remark made before Theorem 2.2 implies that there exists $u_{t} \in S_{r}^{\nu}\left(\Pi_{n}\right)$ for which

$$
\left\|v_{t}(.) \psi^{(0,1)}\left(., x_{0}(.)\right)-u_{t}(.)\right\|_{1}=O(h)
$$

Therefore,

$$
\begin{aligned}
\left\|g_{t}-u_{t}\right\|_{1}= & \int_{0}^{1}\left|m(t, s) k(t-s) \psi^{(0,1)}\left(s, x_{0}(s)\right)-u_{t}(s)\right| d s \\
\leq & \int_{0}^{1}\left|m(t, s) k(t-s) \psi^{(0,1)}\left(s, x_{0}(s)\right)-v_{t}(s) \psi^{(0,1)}\left(s, x_{0}(s)\right)\right| d s \\
& +\int_{0}^{1}\left|v_{t}(s) \psi^{(0,1)}\left(s, x_{0}(s)\right)-u_{t}(s)\right| d s \\
\leq & \left\|k_{t}-v_{t}\right\|_{1}\left\|\psi^{(0,1)}\left(., x_{0}(.)\right)\right\|_{\infty}+\left\|v_{t}(.) \psi^{(0,1)}\left(., x_{0}(.)\right)-u_{t}\right\|_{1} \\
= & O\left(h^{\alpha}\right)+O(h)=O\left(h^{\alpha}\right) .
\end{aligned}
$$

Now, applying Theorem 3.3 with $q=1, p=\infty$, and $X_{n}=S_{r}^{\nu}\left(\Pi_{n}\right)$, we conclude that

$$
\begin{aligned}
\left\|x_{0}-x_{n}^{\prime}\right\|_{\infty} & \leq C\left\{\left\|x_{0}-P_{n} x_{0}\right\|_{\infty}^{2}+\inf _{u \in S_{r}^{\nu}\left(\Pi_{n}\right)}\left\|g_{t}-u_{t}\right\|_{1}\left\|x_{0}-P_{n} x_{0}\right\|_{\infty}\right\} \\
& =O\left(h^{\alpha+\gamma}\right)+O\left(h^{2 \gamma}\right)=O\left(h^{2 \gamma}\right) .
\end{aligned}
$$

The proof is complete.

Next, we apply Theorem 3.3 to equation (1.1) with kernels given by (2.21) and (2.22) and use $X_{n}=S_{r}^{\nu}\left(\Pi_{n}^{\gamma}\right)$ as approximate spaces, where $S_{r}^{\nu}\left(\Pi_{n}^{\gamma}\right)$ of splines with nonuniform knots is defined as in Section 2 such that $r \geq 2$ and $\nu=1$.

Theorem 3.6 Let $x_{0}$ be an isolated solution of (1.1) with weakly singular kernels given by (2.21) and (2.22). Let $x_{n}$ be the unique solution of (2.5) in $B\left(x_{0}, \delta_{1}\right)$, and $x_{n}^{\prime}$ be defined by the iterated scheme (3.1). Assume that 1 is not an eigenvalue of $(K \Psi)^{\prime}\left(x_{0}\right)$ and that the hypotheses of Theorem 2.4 are satisfied with $\mu \geq 1$. Also assume that $\psi^{(0,1)}\left(\cdot, x_{0}(\cdot)\right)$ is of Type $(\alpha, r,\{0,1\})$ for $\alpha>0$ whenever $x_{0}$ is of the same type. Then

$$
\left\|x_{0}-x_{n}^{\prime}\right\|_{\infty}=O\left(\frac{1}{n^{\alpha+r}}\right) .
$$


Proof: The proof of this theorem is similar to that of Theorem 3.5. We apply Theorem 3.3 with $q=1, p=\infty$ and $X_{n}=S_{r}^{\nu}\left(\Pi_{n}^{\gamma}\right)$. By Rice (1969), we have $\left\|x_{0}-P_{n} x_{0}\right\|_{\infty}=O\left(\frac{1}{n^{r}}\right)$. It can be proved that there exists $u \in S_{r}^{\nu}\left(\Pi_{n}^{\gamma}\right)$ such that $\left\|g_{t}-u\right\|_{1}=O\left(\frac{1}{n^{\alpha}}\right)$. From this, the result of this theorem follows.

As the last application of Theorem 3.3, we consider equation (1.1) with kernels having singularity at the four corners of the square $[0,1] \times[0,1]$, a problem that arises from boundary integration for the harmonic Dirichlet problem in plane domains with corners (see Kress (1990)). In the following theorem, we assume $k_{s}(t)=k(t, s)$ is of Type $(\alpha, \mu,\{0,1\})$, for $\alpha>0$, and $k_{t}(s)=k(t, s)$ is of Type $(\alpha, \mu,\{0,1\})$, for $\alpha>-1$, e.g., $k(t, s)=m(t, s) \sqrt{t}$, and $k(t, s)=m(t, s) \frac{1}{\sqrt{1-s}}$, etc., with $m(t, s)$ smooth, and assume $f$ is of Type $(\beta, \mu,\{0,1\})$, for $\alpha, \beta>0$ and a positive integer $\mu$. It is not difficult to prove that an isolated solution $x_{0}$, of the corresponding equation (1.1), is of Type $(\gamma, \mu,\{0,1\})$, where $\gamma=\min \{\alpha, \beta\}$ if $\alpha>0$ and $\gamma=\min \{\alpha+1, \beta\}$ if $-1<\alpha<0$ by modifying the proofs of theorems in Kaneko, Noren and Xu (1990). We again let $q=\frac{r}{\gamma}$ and define the Galerkin subspace $S_{r}^{\nu}\left(\Pi_{n}^{\gamma}\right)$ as in Section 2 with $r=1$ and $\nu=0$, and $r \geq 2$ and $\nu \in\{0,1\}$, where partition $\Pi_{n}^{\gamma}$ is defined as in (2.23). The following theorem describes the order of convergence of the Galerkin approximation $x_{n}$ and that of superconvergence of the iterated Galerkin approximation $x_{n}^{\prime}$. To the best of our knowledge, this result is not known in the literature even for Fredholm integral equations of the second kind.

Theorem 3.7 Let $x_{0}$ be an isolated solution of (1.1) with kernels of the type defined in the paragraph preceding this theorem. Let $x_{n}$ be the unique solution of (2.5) in $B\left(x_{0}, \delta_{1}\right)$, and $x_{n}^{\prime}$ be defined by the iterated scheme (3.1). Assume that 1 is not an eigenvalue of $(K \Psi)^{\prime}\left(x_{0}\right)$ and that $f$ is of Type $(\beta, r,\{0,1\})$. Also assume that $\psi^{(0,1)}\left(\cdot, x_{0}(\cdot)\right)$ is of Type $(\gamma, r,\{0,1\})$ whenever $x_{0}$ is of the same type. Then,

$$
\left\|x_{0}-x_{n}\right\|_{\infty}=O\left(\frac{1}{n^{r}}\right)
$$

and

$$
\left\|x_{0}-x_{n}^{\prime}\right\|_{\infty}=O\left(\frac{1}{n^{2 r}}\right)
$$

Proof: We present the proof for case when $\alpha>0$, since the proof for the other case is similar. The proof of the first estimate is similar to that for Theorem 2.6. Thus, we omit the details. Since $P_{n}$ in this theorem is defined to be the Galerkin projection from $C[0,1]$ onto $S_{r}^{\nu}\left(\Pi_{n}^{\gamma}\right)$, where $\gamma=\min \{\alpha, \beta\}$, and since $x_{0}$ is of $\operatorname{Type}(\gamma, r,\{0,1\})$, we have $\left\|x_{0}-P_{n} x_{0}\right\|_{\infty}=O\left(\frac{1}{n^{r}}\right)$. Meanwhile, since $k_{t}(s)=k(t, s)$ is of Type $(\alpha, r,\{0,1\})$ and $\gamma \leq \alpha$, we find that $k_{t}(s)=k(t, s)$ is also of Type $(\gamma, r,\{0,1\})$. By the assumption on $\psi^{(0,1)}$, we conclude that $\psi^{(0,1)}\left(., x_{0}().\right)$ is of 
Type $(\gamma, r,\{0,1\})$. Hence, $k(t,.) \psi^{(0,1)}\left(., x_{0}().\right)$ is of Type $(\gamma, r,\{0,1\})$. It follows that

$$
\inf _{u \in S_{r}^{\nu}\left(\Pi_{n}^{\gamma}\right)}\left\|k(t, .) \psi^{(0,1)}\left(., x_{0}(.)\right)-u\right\|_{1}=O\left(\frac{1}{n^{r}}\right) .
$$

Therefore, the result of this theorem follows from Theorem 3.3. The proof is complete.

\section{The Iterated Galerkin-Kantorovich Method}

In this section, we extend the classical Kantorovich regularization and the iterated GalerkinKantorovich method for Fredholm integral equations of the second kind to Hammerstein equations. These extensions will be made on equations with both singular kernels and singular forcing terms. The superconvergence of the corresponding iterated solution is also investigated.

In equation (2.4) we put

$$
z=K \Psi x
$$

so that

$$
x=f+z .
$$

Upon applying $K \Psi$ on both sides of (4.2), we obtain

$$
z=K \Psi(f+z)
$$

Now we define operators by $\Psi_{0}(x)(t) \equiv \psi(t, x(t))$, and

$$
\Psi_{1}(x)(t) \equiv \Psi_{0}(f+x)(t)-\Psi_{0}(f)(t)
$$

In addition, define $f_{1}$ by

$$
f_{1}(t) \equiv K \Psi_{0}(f)(t)=\int_{a}^{b} k(t, s) \psi(s, f(s)) d s .
$$

From (4.4), we have $K \Psi_{0}(f+z)(t)=K \Psi_{1}(z)(t)+K \Psi_{0}(f)(t)$ so that (4.3) becomes

$$
z-K \Psi_{1}(z)=K \Psi_{0}(f) \equiv f_{1}
$$

Equation (4.6) will be called the "regularized" equation for the original Hammerstein equation (1.1). It is interesting to note that

$$
\left|\Psi_{1}\left(x_{1}\right)(t)-\Psi_{1}\left(x_{2}\right)(t)\right|=\left|\Psi_{0}\left(f+x_{1}\right)(t)-\Psi_{0}\left(f+x_{2}\right)(t)\right| \leq C_{1}\left|x_{1}(t)-x_{2}(t)\right| .
$$

Thus, $\Psi_{1}$ is also Lipschitz continuous with the same Lipschitz constant $C_{1}$ as one for $\Psi_{0}$. Hence the solvability of equation (4.6) is guaranteed by the solvability of the original equation (1.1). 
The Galerkin method described in Section 2 is now applied to equation (4.6). Namely, we find $z_{n} \in X_{n}$ that satisfies

$$
z_{n}-P_{n} K \Psi_{1} z_{n}=P_{n} f_{1}
$$

The Galerkin-Kantorovich regularization solution for (1.1) is now given by

$$
x_{n}^{K}=f+z_{n} .
$$

Note that $x_{n}^{K}$ inherits the singularity of $f$. From equations (4.2) and (4.8), we have $x-x_{n}^{K}=z-z_{n}$. Since $z, z_{n} \in C[0,1]$, we see that $x-x_{n}^{K} \in C[0,1]$, although neither $x$ nor $x_{n}^{k}$ may be in $C[0,1]$. Denote $T_{n} z_{n} \equiv P_{n} f_{1}+P_{n} K \Psi_{1} z_{n}$ and $T z \equiv f_{1}+K \Psi_{1} z$.

Theorem 4.1 Let $x_{0}$ be an isolated solution of equation (1.1) such that $z_{0}=K \Psi_{0} x_{0} \in C[0,1]$. Assume that 1 is not an eigenvalue of the linear operator $\left(K \Psi_{1}\right)^{\prime}\left(z_{0}\right)$. Then equation (4.7) has a unique solution $z_{n} \in B\left(z_{0}, \delta\right)$ for some $\delta>0$ and for sufficiently large $n$. Moreover, there exists a constant $0<q<1$, independent of $n$, such that

$$
\frac{\alpha_{n}}{1+q} \leq\left\|x_{n}^{K}-x_{0}\right\|_{\infty} \leq \frac{\alpha_{n}}{1-q}
$$

where $x_{n}^{K}=f+z_{n}$ and

$$
\alpha_{n}=\left\|\left(I-T_{n}^{\prime}\left(z_{0}\right)\right)^{-1}\left(T_{n}\left(z_{0}\right)-T\left(z_{0}\right)\right)\right\|_{\infty}
$$

Finally,

$$
E_{n}\left(z_{0}\right) \leq\left\|x_{0}-x_{n}^{K}\right\|_{\infty} \leq C E_{n}\left(z_{0}\right)
$$

where $E_{n}\left(z_{0}\right)=\inf _{y \in X_{n}}\left\|y-z_{0}\right\|_{\infty}$ and $C$ is a constant independent of $n$.

Proof: The inequalities (4.9) follows again from Theorem 2 of Vainikko (1967). Also it is noted that

$$
z_{0}-z_{n}=x_{0}-x_{n}^{K}
$$

Since $z_{n} \in X_{n}$, (4.10) holds and $E_{n}\left(z_{0}\right) \leq\left\|z_{0}-z_{n}\right\|_{\infty}=\left\|x_{0}-x_{n}^{K}\right\|_{\infty}$. This gives the first inequality in (4.11). Since $T_{n}\left(z_{0}\right)-T\left(z_{0}\right)=P_{n}\left(f_{1}-K \Psi_{1} z_{0}\right)-z_{0}=P_{n} z_{0}-z_{0}$, we find

$$
\left\|z_{n}-z_{0}\right\|_{\infty} \leq \frac{\left\|\left(I-T_{n}^{\prime}\left(z_{0}\right)\right)^{-1}\right\|_{\infty}\left\|T_{n}\left(z_{0}\right)-T\left(z_{0}\right)\right\|_{\infty}}{1-q}=\frac{\left\|\left(I-T_{n}^{\prime}\left(z_{0}\right)\right)^{-1}\right\|_{\infty}}{1-q}\left\|P_{n} z_{0}-z_{0}\right\|_{\infty} .
$$

Also for $u \in X_{n}$,

$$
\left\|z_{0}-P_{n} z_{0}\right\|=\left\|z_{0}-u-P_{n}\left(z_{0}-u\right)\right\| \leq\left(1+\left\|P_{n}\right\|\right)\left\|z_{0}-u\right\| .
$$

Therefore, we have $\left\|x_{0}-x_{n}^{K}\right\| \leq C E_{n}\left(z_{0}\right)$ where $C$ is a constant independent of $n$. 
We next consider the iterated Kantorovich method and investigate its superconvergence property. Assume that $z_{0}$ is an isolated solution of (4.6) and $z_{n}$ is the unique solution of (4.7) in the sphere $B\left(z_{0}, \delta\right)$ for some $\delta>0$. Define

$$
z_{n}^{\prime}=K \Psi_{1}\left(z_{n}\right)+f_{1}
$$

and $x_{n}^{K^{\prime}}=f+z_{n}^{\prime}$. The element $x_{n}^{K^{\prime}}$ is called the iterated Galerkin-Kantorovich approximate solution of equation (1.1). Applying $P_{n}$ to both sides of (4.13) gives

$$
P_{n} z_{n}^{\prime}=P_{n} K \Psi_{1}\left(z_{n}\right)+P_{n} f_{1}
$$

Again, by using (4.7), we have $P_{n} z_{n}^{\prime}=z_{n}$. Upon substituting this equation into (4.13), we find that $z_{n}^{\prime}$ satisfies the following new Hammerstein equation $z_{n}^{\prime}=K \Psi_{1} P_{n} z_{n}^{\prime}+f_{1}$. In view of the fact that $\Psi_{1}$ is Lipschitz continuous with the same Lipschitz constant as one for $\Psi_{0}$, the same proofs given for Theorems 3.1, 3.2 and 3.3 can be applied to $S_{n} \equiv K \Psi_{1} P_{n}+f_{1}$ to obtain the following theorem. Here $\delta_{1}$ is chosen as in Section 3. As in Theorem 3.3, the assumption that $\left\|(K \Psi)^{\prime}\left(x_{0}\right)\left(I-P_{n}\right)\right\|_{\infty} \rightarrow 0$ as $n \rightarrow \infty$ is no longer needed.

Theorem 4.2 Let $x_{0}$ be an isolated solution of equation (1.1) such that $z_{0}=K \Psi_{0} x_{0} \in C[0,1]$. Let $z_{n}$ be the unique solution of equation (4.7) in the sphere $B\left(z_{0}, \delta_{1}\right)$. Let $x_{n}^{K^{\prime}}$ be the corresponding iterated Galerkin- Kantorovich approximate solution. Assume that 1 is not an eigenvalue of $\left(K \Psi_{1}\right)^{\prime}\left(z_{0}\right)$. Then, for all $1 \leq p \leq \infty$,

$$
\left\|x_{0}-x_{n}^{K^{\prime}}\right\| \leq C\left\{\left\|z_{0}-P_{n} z_{0}\right\|_{\infty}^{2}+\sup _{0 \leq t \leq 1} \inf _{u \in X_{n}}\left\|k(t, \cdot) \psi_{1}^{(0,1)}\left(\cdot, z_{0}(\cdot)\right)-u\right\|_{q}\left\|z_{0}-P_{n} z_{0}\right\|_{p}\right\},
$$

where $\frac{1}{p}+\frac{1}{q}=1$.

Results parallel to Theorems 3.4 - 3.7, for smooth and weakly singular kernels can be obtained also by using Theorem 4.2, for the iterated Kantorovich method. The iterated Kantorovich regularization method for the Fredholm equations of the second kind was investigated by Sloan (1984).

\section{$5 \quad$ Numerical Examples}

In this section, some numerical examples are given to illustrate the theory established in the previous sections.

Example 1: Consider

$$
x(t)-\int_{0}^{1} \frac{x^{2}(s)}{\sqrt{|t-s|}} d s=f(t), \quad 0 \leq t \leq 1,
$$


where $f$ is selected so that $x(t)=\sqrt{t}$ is the solution. The splines of orders $1(q=2)$ and $2(q=4)$ with knots defined by equation (2.23) in terms of $q$, are used in computations. To establish the Galerkin matrix, it is required to compute the integral of the form

$$
\int_{t_{i-1}}^{t_{i}} \int_{t_{j-1}}^{t_{j}} \frac{\varphi_{i}(s) \varphi_{j}(t)}{\sqrt{|t-s|}} d t d s
$$

where $\varphi_{i}^{\prime}$ s are respective B-splines of the above mentioned spline space. It can be proved that $\varphi_{i}(s) \int_{t_{j-1}}^{t_{j}} \frac{\varphi_{j}(t)}{\sqrt{|t-s|}} d t$ belongs to Type $\left(\frac{1}{2}, k,\left\{t_{j-1}, t_{j}\right\}\right)$. Consequently, we employ the recently developed Gauss-type quadrature formula of Kaneko and Xu (1994) to approximate integrals (5.2). This brings to our concern the problem of the discrete Galerkin method for Hammerstein equations with weakly singular kernels. This will be dealt with in a future paper. In the ensuing data, $e_{n} \equiv\left\|x-x_{n}\right\|_{\infty}$ and $e_{n}^{\prime} \equiv\left\|x-x_{n}^{\prime}\right\|_{\infty}$ were approximated respectively by

$$
\max \left\{\left|x\left(\frac{i}{100}\right)-x_{n}\left(\frac{i}{100}\right)\right|: i=0,1, \cdots, 100\right\}
$$

and

$$
\max \left\{\left|x\left(\frac{i}{100}\right)-x_{n}^{\prime}\left(\frac{i}{100}\right)\right|: i=0,1, \cdots, 100\right\}
$$

Data 1. $(q=2)$

$\begin{array}{rcccc}n & e_{n} & \text { decay exp. } & e_{n}^{\prime} & \text { decay exp. } \\ & & & & \\ 16 & 1.60 D-2 & & 3.01 D-3 & \\ 32 & 7.26 D-3 & 1.14 & 9.10 D-4 & 1.73 \\ 64 & 3.34 D-3 & 1.12 & 2.88 D-4 & 1.66 \\ 128 & 1.64 D-3 & 1.03 & 9.50 D-5 & 1.60\end{array}$

Data 2. $(q=4)$

$\begin{array}{rcccc}n & e_{n} & \text { decay exp. } & e_{n}^{\prime} & \text { decay exp. } \\ & & & & \\ 16 & 4.01 D-3 & & 8.04 D-4 & \\ 32 & 9.93 D-4 & 2.01 & 1.30 D-4 & 2.61 \\ 64 & 2.46 D-4 & 2.01 & 2.28 D-5 & 2.51 \\ 128 & 6.06 D-5 & 2.02 & 3.90 D-6 & 2.55\end{array}$


It can be seen clearly that the iterated Galerkin approximation has superconvergence by an order $\frac{1}{2}$.

Example 2. To illustrate the use of theorem 3.7, we consider

$$
x(t)-\int_{0}^{1} \frac{x^{2}(s)}{\sqrt[3]{s}} d s=f(t), \quad 0 \leq t \leq 1,
$$

where $f$ is selected so that $x(t)=\sqrt{t}$ is the solution of equation (5.3). As in the first example, the splines of orders 1 and 2 are used. Since the solution is of Type $\left(\frac{1}{2}, k,\{0,1\}\right)$ for any positive integer $k$, the partition is formed according to $\alpha=\frac{1}{2}$.

Data 1. $(q=2)$

$\begin{array}{rcccc}n & e_{n} & \text { decay exp. } & e_{n}^{\prime} & \text { decay exp. } \\ & & & & \\ 16 & 1.12 D-2 & & 2.10 D-3 & \\ 32 & 5.15 D-3 & 1.12 & 5.21 D-4 & 2.01 \\ 64 & 2.22 D-3 & 1.21 & 1.30 D-4 & 2.00 \\ 128 & 1.08 D-3 & 1.04 & 3.25 D-5 & 2.00\end{array}$

Data 2. $(q=4)$

$n \quad e_{n} \quad$ decay exp. $\quad e_{n}^{\prime} \quad$ decay exp.

$\begin{array}{rrrrr}16 & 3.12 D-3 & & 5.12 D-4 & \\ 32 & 7.53 D-4 & 2.05 & 3.05 D-5 & 4.07 \\ 64 & 1.74 D-4 & 2.11 & 1.85 D-6 & 4.04 \\ 128 & 4.26 D-5 & 2.03 & 1.14 D-7 & 4.02\end{array}$

The iteration process doubles the rate of convergence.

ACKNOWLEDGEMENT: The authors would like to thank Professor K. Atkinson and the referee who made many useful suggestions that improved this paper.

\section{References}

1. P. M. Anselone, Collectively compact operator approximation theory and applications to Integral Equations, Prentice-Hall, Englewood Cliffs, N.J., 1971. 
2. K. E. Atkinson, A Survey of Numerical Methods for the Solution of Fredholm Integral Equations of the Second Kind, SIAM, 1976.

3. K. E. Atkinson, A survey of numerical methods for solving nonlinear integral equations, $J$. Int. Equ. Appl. 4 (1992), 15-46.

4. K. E. Atkinson and J. Flores, The discrete collocation method for nonlinear integral equations, Report on Computational Mathematics, No. 10, the University of Iowa, 1991.

5. K. E. Atkinson, I. Graham and I. Sloan, Piecewise continuous collocation for integral equations, SIAM J. Numer. Anal. 20 (1983), 172-186.

6. K. E. Atkinson and F. Potra, Projection and iterated projection methods for nonlinear integral equations, SIAM J. Numer. Anal. 24 (1987), 1352-1373.

7. C. de Boor, A bound on the $L_{\infty}$ norm of $L_{2}$-approximation by splines in terms of a global mesh radio. Math. Comp. 30 (1976), 765-771.

8. J. P. Delahaye, Sequence Transformations, Springer-Verlag, Berlin Heidelberg, 1988.

9. S. Demko, Splines approximation in Banach function spaces, in Theory of Approximation with Applications (Law, A. G. \& Sahney, B. N., Eds), pp. 146-154, New York, Academic Press, 1976.

10. R. A. De Vore, Degree of approximation, in Approximation Theory II (Lorentz, G. G., Chui, C. K. \& Schumaker, L. L., Eds) pp. 117-161, New York, Academic Press, 1976.

11. J. Douglas, T. Dupont, and L. Wahlbin, Optimal $L_{\infty}$ error estimates for Galerkin approximations to solutions of two point boundary value problems, Math. Comp. 29 (1975), 475-483.

12. I. Graham, Galerkin Methods for second kind integral equations with singularities, Math. Comp. 39 (1982), 519- 533.

13. I. Graham, S. Joe and I. Sloan, Iterated Galerkin versus iterated collocation for integral equations of the second kind, IMA J. Numer. Anal. 5 (1985), 355-369.

14. H. Kaneko, R. Noren and Yuesheng Xu, Regularity of the solution of Hammerstein equations with weakly singular kernels, Int. Eqs. Op. Thy. 13 (1990), 660-670. 
15. H. Kaneko, R. Noren and Yuesheng Xu, Numerical solutions for weakly singular Hammerstein equations and their superconvergence, J. Int. Eqs. Appl. 4 (1992), 391-407.

16. H. Kaneko and Yuesheng Xu, Degenerate kernel method for Hammerstein equations, Math. Comp. 56 (1991), 141-148.

17. H. Kaneko and Yuesheng $\mathrm{Xu}$, Gauss-type quadratures for weakly singular integrals and their application to Fredholm integral equations of the second kind, Math. Comp. , 62 (1994), 739-753.

18. L. V. Kantorovich, Functional analysis and applied mathematics, Usp. Mat. Mauk. 3 (1948), 89-185. English Trans. N. B. S. report 1509 (1952).

19. R. Kress, A Nyström method for boundary integral equations in domains with corners, Numer. Math. 58 (1990), 145-161.

20. S. Kumar, A discrete collocation-type method for Hammerstein equation, SIAM J. Numer. Anal. 25 (1988), 328-341.

21. S. Kumar, Superconvergence of a collocation-type method for Hammerstein equations, IMA J. Numer. Anal. 7 (1987), 313-325.

22. S. Kumar and I. H. Sloan, A new collocation-type method for Hammerstein equations, Math. Comp. 48 (1987), 585-593.

23. L. J. Lardy, A variation of Nystrom's method for Hammerstein equations, J. Int. Eqs. 3 (1981), 43-60.

24. J. Rice, On the degree of convergence of nonlinear spline approximation, in Approximation with Special Emphasis on Spline Functions, ed. I. J. Schoenberg, Academic Press, N.Y. (1969), 349-365.

25. C. Scheider, Product integration for weakly singular integral equations, Math. Comp.36 (1981), 207-213.

26. I. H. Sloan, Four variants of the Galerkin methods for integral equations of the second kind, IMA J. Numer. Anal. 4 (1984), 9-17.

27. I. H. Sloan, Improvement by iteration for compact operator equations, Math. Comp. 30 (1976), 758-764. 
28. I. H. Sloan, Superconvergence, in Numerical Solution of integral equations, ed. M. A. Golberg, Plenum Press, N.Y., 1990, 35-70.

29. I. H. Sloan and V. Thomee, Superconvergence of the Galerkin iterates for Integral equations of the second kind, J. Int. Eqs. 9 (1985), 1-23. 22 (1981), 431-438.

30. G. Vainikko, Perturbed Galerkin method and general theory of approximate methods for nonlinear equations, Zh. Vychisl. Mat. Fiz. 7 (1967), 723-751. Engl. Translation, U.S.S.R. Comp. Math. and Math. Phys. 7, No. 4 (1967), 1-41.

31. G. Vainikko and P. Uba, A piecewise polynomial approximation to the solution of an integral equation with weakly singular kernel, J. Austral. Math. Soc. Series B, 22 (1981), 431-438.

32. G. Vainikko, A. Pedas and P. Uba, Methods of Solving Weakly Singular Integral Equations (in Russian), Tartu University, 1984.

33. J. Wimp, Sequence Transformations and their Applications, Academic Press, New York, 1981.

34. E. Zeidler, Nonlinear Functional Analysis and its Applications II/B, Springer-Verlag, New York, 1990. 